\title{
La exclusión social en el Área Metropolitana de Monterrey: procesos sociales y trayectorias vitales
}

\author{
Social exclusion in the Metropolitan Area of Monterrey: \\ social process and life paths \\ Javier José GARCÍA JUSTICIA \\ Universidad de Monterrey \\ jjgjusticia@gmail.com
}

Recibido: $11 / 03 / 2013$

Revisado: 24/05/2013

Aceptado: 20/09/2013

Disponible on line: $27 / 06 / 2014$

\begin{abstract}
Resumen
La superación de la pobreza está bloqueada por las dinámicas de exclusión social. Ésta cuestiona la premisa de la movilidad ascendente. El acoplamiento de factores de exclusión socioeconómica, sociocultural y sociopolítica, aunado a una fuerte segregación espacial, tiende a reforzar, potenciar y perpetuar las formas de pobreza y desigualdad más extremas y persistentes. En el caso específico del Área Metropolitana de Monterrey, los mecanismos que generan principalmente los procesos de exclusión social son los relativos a los ámbitos cultural-simbólico y al político-institucional, reflejados en el debilitamiento de las redes sociales, el no reconocimiento de los derechos sociales y el acceso a la ciudadanía, la discriminación, la dominación cultural y la cultura de la superioridad, aunado a la debilidad de las políticas públicas para hacer frente a las diferentes situaciones de exclusión.
\end{abstract}

Palabras clave: exclusión social, Monterrey, procesos sociales, trayectorias vitales, redes sociales, discriminación, políticas públicas.

\begin{abstract}
The ability to overcome poverty is blocked by the dynamics of social exclusion. This article examines the notion of upward mobility. The linking of the factors of socio-economic, socio-cultural and socio-political exclusion with strong spacial segregation, have the tendency to reinforce, strengthen and perpetuate the most extreme and persistent forms of poverty and inequality. In the particular case of Metropolitan Monterrey, the mechanims that primarily generate the processes of social exclusion are those connected with the cultural-symbolic and political-institutional realms, reflected in the weakening of social networks, the lack of recognition of social rights and access to civic life, discrimination, cultural domination and the culture of superiority joining with the paucity of public policy to cope with the different aspects of exclusion.
\end{abstract}

Keywords: social exclusion, Monterrey, social processes, life paths, social networks, discrimination, public policies.

Referencia normalizada: García Justicia, J. J. (2014): «La exclusión social en el Área Metropolitana de Monterrey: procesos sociales y trayectorias vitales». Cuadernos de Trabajo Social, 27(1): 175-186.

Sumario: Introducción. 1. Aproximación teórica. 2. Posiciones en torno al lugar donde se ubica la exclusión social. 3. Planteamiento metodológico. 4. La dinámica de la exclusión social: procesos y trayectorias vitales. 5. Principales aprendizajes. 6. Conclusión. 7. Referencias bibliográficas.

\section{Introducción}

La pobreza se ha tornado cada vez más compleja, al igual que la sociedad en su conjunto, lo que dificulta encontrar soluciones que permitan reducir sus niveles de modo significativo. La condición de pobreza urbana actual no sólo se define por las carencias de determina- dos segmentos sociales que ocupan las posiciones más bajas de la estructura social, sino también y sobre todo porque son los que están más fuertemente sometidos a los nuevos mecanismos de marginación y exclusión social derivados del capitalismo globalizado, además de que en aquélla se potencian elementos étnicos, 
raciales, culturales, de género, sexuales, ideológicos, religiosos y políticos.

Los estudios sobre pobreza y marginación tradicionalmente se han centrado en apuntar a la persona que las sufre para lograr una intervención adecuada. Sin embargo y sin perder esta perspectiva de intervención, es necesario rescatar el hecho de que estas realidades no se superan poniendo todo el esfuerzo en el que la está sufriendo, sino también en la sociedad en su conjunto. Mientras el foco de intervención sea únicamente la persona que sufre algún tipo de pobreza, marginación y exclusión, nos enfrentaremos a una intervención limitada, insuficiente e incluso vana para su superación. De modo pertinente, señala Castel (1997), que: «El corazón de la problemática de la exclusión no está en dónde encontramos a los excluidos» (p. 108). Hay que buscarla en el control y acaparamiento de los recursos por ciertos grupos sociales que marginan a otros.

Ante este panorama, es necesario un replanteamiento en la forma de enfocar el estudio sobre la pobreza, que deja de ser el pobre como un simple número en una estadística, centrándose en los sujetos en situación de exclusión y en la sociedad que ejerce la fuerza exclusora, es decir, en las barreras que encuentran determinadas personas y colectivos para salir adelante, las fronteras sociales, tanto materiales como sobre todo sociales, culturales y simbólicas que les impiden ser ciudadanos con plenitud de derechos. La exclusión social pone de manifiesto las tendencias dualizadoras y segregadoras del sistema económico, la progresiva polarización social y el riesgo de fragmentación y desintegración de una parte de la sociedad (Rubio y Mortero, 2002), una parte que parece olvidada en América Latina desde hace mucho tiempo.

La pobreza y la exclusión social se producen y reproducen de generación en generación, favorecidas por problemas de naturaleza estructural y social, además de estar reforzada por la socialización en los valores de la cultura de la pobreza, situación que desemboca en un círculo vicioso de pobreza muy resistente y difícil de romper. Buscando factores de génesis del fenómeno multidimensional de la exclusión social se podría reinterpretar como el acoplamiento de varios tipos de exclusiones: la socioeconómica, la sociocultural y la políticoinstitucional. Cuan- do acaece tal acoplamiento, la exclusión tiende a reforzarse adquiriendo formas más extremas y persistentes (Gacitúa, Sojo y Davis, 2000).

La exclusión es tan grave y compleja que, aunque alguna o algunas de estas situaciones redujeran su intensidad, el problema no se erradicaría. Los problemas no son compartimentos estancos, se interrelacionan e imbrican de modo que crean un círculo vicioso y duro del que no es nada fácil escapar. Por otra parte, las personas que viven en esta situación, progresivamente pierden sus capacidades y habilidades sociales, si alguna vez las adquirieron, hecho que retroalimenta nuevamente la situación de partida reforzando la exclusión e impidiendo su participación, en definitiva: no están dentro $\mathrm{y}$ no pueden entrar sin ayuda (IESA-CSIC, 2010).

La exclusión socioeconómica es aquella que está asociada con la desigualdad en la distribución, además del acceso a los bienes, servicios y recursos. Sin embargo, mientras los resultados pueden ser socioeconómicos, o materiales, hay un reconocimiento de que la exclusión puede tener su origen, bien en parte o enteramente, en la marginación cultural simbólica. La exclusión cultural simbólica está asociada con el no reconocimiento de los derechos sociales y el acceso a la ciudadanía, la falta de respeto y la dominación cultural. Los dos tipos de exclusión pueden llevar a una distribución desigual de los recursos que, a su vez, pueden contribuir a la marginación financiera y material y a la dificultad de acceso a los recursos, a los bienes y a las redes sociales que conlleva la estigmatización y la denigración. El enfocarse únicamente en las consecuencias relativas a las desventajas de nacimiento, trasmitidas de generación en generación, pone el foco de atención en los individuos y sus circunstancias personales, pero es una aproximación que sirve para generar patologías en los individuos más que para promover la comprensión de los procesos sociales que contribuyen a la marginación y, por lo tanto, la visión es limitada (Ward, 2009).

Así pues, el objetivo principal del artículo es conocer, comprender y evaluar los mecanismos que generan principalmente los factores de exclusión social en el Área Metropolitana de Monterrey. Las preguntas que surgen en torno a un fenómeno de este calibre, y que preten- 
den responderse en el presente artículo, son las siguientes: ¿Cuáles son los mecanismos y estructuras que producen exclusión social? ¿Cuáles son los factores que activan los circuitos de exclusión social? ¿Cuáles son las fronteras materiales, sociales, culturales y simbólicas que limitan el acceso a los espacios y recursos sociales más valorados en el Area Metropolitana de Monterrey? ¿Quiénes son los grupos o las personas más afectados por la exclusión en este espacio urbano? ¿Qué significa ser excluido en esta ciudad? ¿Qué es aquello que actúa como expulsor social en esta urbe?

\section{Aproximación teórica}

En el presente artículo se entenderá por exclusión social el proceso social de desintegración, expulsión, separación o inaccesibilidad que impide a un grupo de ciudadanos tener acceso, o que éste sea limitado, a los derechos políticos y económicos, las oportunidades vitales fundamentales y los recursos socialmente valiosos de una sociedad determinada. Como consecuencia de ello, los individuos o las comunidades no pueden pertenecer y participar plenamente en la sociedad porque sus vínculos sociales se debilitan o se rompen. Este hecho apunta a un claro deterioro o una ausencia de los derechos económicos, sociales y políticos e impide un pleno desarrollo de las personas y sus capacidades.

Entre las características clave podemos destacar que es un fenómeno estructural, dinámico, multifactorial y multidimensional, y susceptible de politización (Subirats y Gomà, 2003; Raya, 2004; Subirats, 2006; Hernández, 2008; Mira, 2008; ICGP, 2010); está ubicado en la sociedad postindustrial y vinculado con los procesos de cambio social, económico, demográfico, espacial, cultural y político. Estas transformaciones conllevan como contracara el aumento de la pobreza, la marginación y la exclusión social.

En el análisis de las diferentes dimensiones que mide la exclusión social, además de las ya explicitadas anteriormente, que se refieren a los aspectos socioeconómico, sociocultural y sociopolítico, cabe señalar igualmente los factores de tiempo y de espacio. La exclusión social es la acumulación en el tiempo y el espacio de riesgos específicos que dificultan o impiden la realización de ciertos derechos (ci- viles, económicos, sociales, culturales y políticos) y la integración del grupo social afectado con su medio o la sociedad. La dimensión temporal indica que la exclusión es el resultado de un proceso histórico, de su evolución y de la acumulación de factores de riesgo en circunstancias históricas concretas. La dimensión espacial o territorial no sólo es que la exclusión ocurre en un determinado espacio, sino que existen fenómenos espaciales que se constituyen en factores de riesgo generadores de exclusión (integración a mercados, sistemas de producción, patrones de asentamiento, etc.).

Entre las diferentes dimensiones que abarca el concepto se puede hablar también de un plano objetivo y otro subjetivo. El plano objetivo considera las condiciones efectivas en que se encuentran los sujetos. La exclusión permite identificar factores de riesgo objetivos, tales como la ubicación espacial, la dificultad o imposibilidad de acceder al mercado laboral entre otros. Por otro lado, la exclusión social introduce el análisis de la construcción social que hacen los sujetos, tanto en cómo se perciben a sí mismos como a los mecanismos de acción que se ponen en marcha basados en esa interpretación del factor de riesgo, hecho que se verá con mayor detalle en el apartado del capital social y las redes sociales.

Al hablar de exclusión deviene insoslayable el tema de la desigualdad. Algo básico que se necesita tener en cuenta es que la exclusión social, en tanto que representa la forma extrema de las desigualdades sociales, remite a una comprensión relacional de la sociedad basada en el poder; al contrario que la pobreza que define las carencias en términos de un cierto estándar de bienestar, remitiendo así a una comprensión no relacional, ya que solo busca identificar grupos de población pobres, necesario en un principio en toda investigación, pero siendo una perspectiva limitada cuando se quiere ahondar en las causas de la misma.

En el contexto latinoamericano es básico destacar los nexos entre desigualdad y exclusión social, principalmente entre las dos principales modalidades de producción de desigualdad social en este continente: la explotación y el acaparamiento de oportunidades (Tilly, 1999).

Para superar la pobreza es necesario movilizar acciones, mientras que la exclusión señala 
a las barreras sociales que dificultan el logro de tal cometido. La exclusión cuestiona la premisa de la movilidad ascendente y propone que hay un bloqueo en la superación de la pobreza.

En las sociedades con grandes disparidades se pierde la confianza entre sus integrantes; con las desigualdades tan pronunciadas baja la identificación y la desconfianza se refuerza. En un contexto así aunado a los altos grados de marginación, los altos grados de corrupción pública y privada, la escasez de las acciones políticas y el debilitamiento de las redes sociales, hay un aumento de los resentimientos al igual que de la frustración, sobre todo cuando se piensa que la riqueza no es legítima, caldo de cultivo perfecto para el conflicto social.

\section{Posiciones en torno al lugar donde se ubi- ca la exclusión social}

Un asunto básico en todo análisis de la exclusión social es el de establecer los límites en el continuo inclusión-vulnerabilidad-exclusión. No se puede olvidar que hay diferentes formas de estar dentro y fuera del sistema. Los excluidos pueden estar fuera de la sociedad o dentro, o en transición en zonas que van desde «dentro» hasta «fuera» (llamadas también zonas de vulnerabilidad).

\subsection{Representación gráfica}

Basándose en la propuesta de Castel (1997), una de las formas gráficas más usadas para representar estos procesos de exclusión social es el Diagrama de Lexis, ya que permite relacionar mediante el eje de abscisas y ordenadas los tres espacios sociales con el calendario o edad de un sujeto (Hernández Pedreño, 1999, 2008; Pérez Yruela, Rodríguez Cabrero y Trujillo Carmona, 2002, 2004) (véase Figura 1). Existe una zona de integración, seguridad o estabilidad, seguida de una zona de vulnerabilidad, precariedad o inestabilidad, y una zona de exclusión o marginación.

La zona de inclusión es un espacio social conformado por personas u hogares cuyas condiciones de vida les permiten desarrollarse y formar parte de la sociedad, vivir según estándares considerados normales, incluso por encima de ellos y pueden ejercer plenamente sus derechos de ciudadanía.

La zona de vulnerabilidad es una instancia intermedia entre la inclusión y la exclusión en

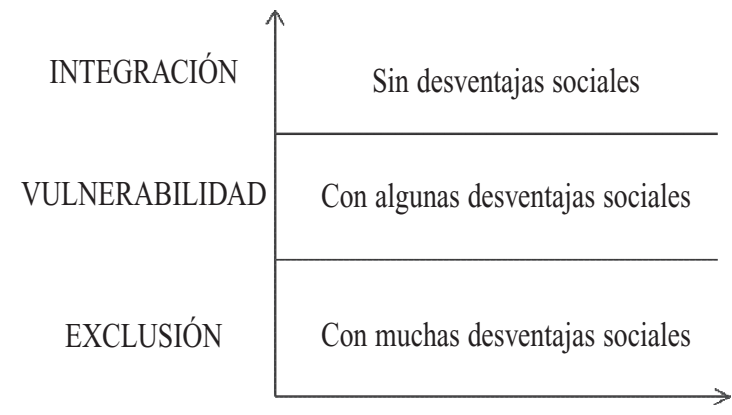

Figura 1. Representación gráfica de los tres espacios sociales.

Fuente: Castel (1997).

donde se generan situaciones de precariedad, fragilidad y debilidad que impiden a un individuo o grupo particular lograr una satisfacción plena o parcial de una necesidad o el acceso a un bien social (Castel, 1997). Una acumulación de vulnerabilidades podría conducir a la exclusión social.

En la zona de exclusión social se clasifican aquellos hogares o personas que se encuentran en los límites o fuera de los mismos, experimentan problemas en todos los ámbitos que configuran el bienestar social, de modo que no pueden vivir según los estándares de referencia, ni pueden ejercer sus derechos de ciudadanía. La exclusión social se vería como una situación de fuerte irreversibilidad que afecta a un porcentaje moderado de población, pero que, no obstante, es susceptible de intervención social. En la exclusión absoluta se encuentran aquellos hogares o personas que acumulan problemas importantes en todos los ámbitos de análisis.

Al aplicar este modelo a la realidad latinoamericana en general, y a la mexicana en concreto, se consideró pertinente añadir un cuarto espacio social —inclusión débil- a los tres clásicos (véase Figura 2). Se tomó esta decisión por la debilidad que existe en un tipo de inclusión. Son personas a las que aparentemente no se les puede considerar vulnerables ni excluidas, pero su situación social les mantiene en un espacio muy débil entre la inclusión y la vulnerabilidad. Estas debilidades giran en torno a varios campos, especialmente el relacionado con el mundo laboral, debido a la inestabilidad de los trabajos y la polarización de los salarios. Al poseer una fuente de trabajo existe el acceso a determinados servicios de 


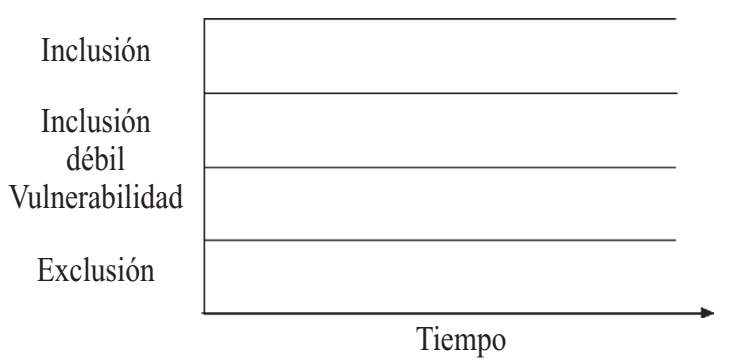

Figura 2. Propuesta personalizada de la representación gráfica de los cuatro espacios sociales para la investigación.

Fuente: Elaboración propia.

salud, vivienda, educación, etc., lo que podría determinar que esa persona está incluida. Sin embargo, la pérdida súbita del mismo haría que se disiparan esas prestaciones y al no contar con unas políticas públicas sólidas de apoyo y poseer una débil capacidad de ahorro, le haría caer automáticamente en una situación de vulnerabilidad o exclusión, si se mantuviera en el tiempo dicha situación. A este hecho se aunaría la falta —o debilidad — de las redes de apoyo, o el que éstas también estuvieran en situaciones similares, que fueran principalmente redes que han sido castigadas por las crisis continuas o bien por estar en situaciones de pobreza permanente intergeneracional.

\section{Planteamiento metodológico}

Para capturar la multidimensionalidad de la exclusión social es necesario combinar diferentes ámbitos relativos a las dimensiones económica, laboral, sanitaria, educativa, habitacional, relacional, político-institucional y contextual; además de un número amplio de factores e indicadores, tanto generadores como potenciadores de la exclusión social.

La combinación de técnicas cuantitativas y cualitativas es muy usual en los estudios científicos sociales, especialmente en los desarrollados en la primera década del siglo XXI en torno al fenómeno de la exclusión social (Pérez Yruela et al., 2002; Subirats y Gomà, 2003; Raya, 2004; Subirats et al., 2005; Ruiz de Olabuénaga, 2007; Mira, 2008; Olaz Capitán, 2008; Hernández 2008; Instituto Catalán de Gobierno y Políticas Públicas - ICGP-, 2010; Instituto de Estudios Sociales Avanzados del Centro Superior de Investigaciones Científicas -IESA/CSIC-, 2010). La complementariedad de técnicas es cada vez más usual, y necesaria, en el estudio de la exclusión social, poniendo de manifiesto la riqueza informativa que se obtiene al combinar técnicas de diverso enfoque.

No obstante, el presente escrito se inclinará en reportar los datos obtenidos mediante diferentes técnicas cualitativas. En cada contexto social, los factores que generan inclusión-exclusión son distintos y, como consecuencia, sería en cada contexto espaciotemporal donde cabría identificar tanto los factores que actúan excluyendo, como las personas que se encontrarían en situación de exclusión, de ahí que un enfoque cuantitativo sería una limitación para investigaciones de este talante, se optó por un acercamiento a las personas para valorar su situación a partir de sus propios relatos personales para conocer la interpretación y el significado que conceden a sus vivencias personales, además de dimensionar su situación en el contexto en el que viven y con una visión histórica. Además de los factores objetivos y esperables de exclusión social, como son el ingreso, la cualificación, el empleo, la vivienda y la salud, se unió la importancia central del componente relacional, cultural y político-institucional de la exclusión. La exclusión puede convertirse en una cultura propia, un modo de vida asumido por la persona del que resulta difícil salir (Mira, 2008).

Entre la gran variedad de técnicas cualitativas que existen para aproximarse a la realidad se optó por dos herramientas: 17 entrevistas en profundidad a personas en situación de exclusión para la realización de sus historias de vida, y 2 grupos de discusión con informantes cualificados. Se escogieron estos enfoques por su reconocida validez científica y porque además ayudan a interpretar los fenómenos desde un punto de vista más integral, pudiendo así analizar los momentos críticos y puntos de inflexión en la trayectoria vital de las personas seleccionadas para la presente investigación, es decir, aquellos donde coinciden uno o más acontecimientos de gran trascendencia; los puntos donde se inician la acumulación de ventajas o desventajas sociales.

El estudio cualitativo se desarrolló en tres fases: la primera, en la que se realizó el primer grupo de discusión con 10 informantes cualificados. En este espacio se determinaron los perfiles de las personas que se habían de entrevis- 


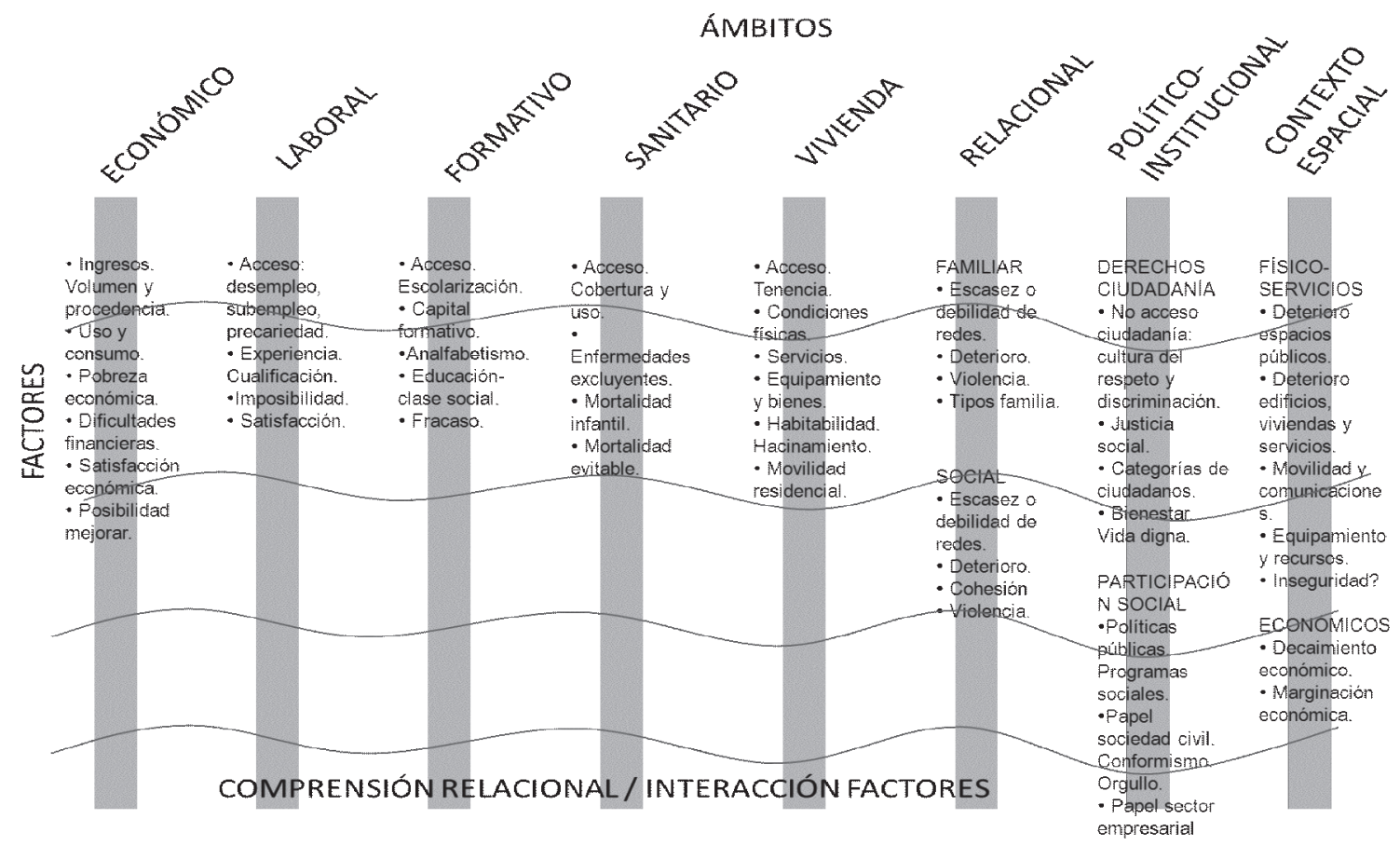

Figura 3. Ámbitos y factores de investigación.

Fuente: Elaboración propia.

tar para las historias de vida al igual que se seleccionaron los ámbitos y factores de investigación pertinentes (véase Figura 3).

La selección de los informantes cualificados se realizó con un triple criterio, por un lado que fueran expertos reconocidos dentro de su campo de trabajo; por otro, que realizaran o hubieran realizado un trabajo directo con personas en situación de vulnerabilidad o exclusión durante un periodo de tiempo largo para que conocieran la realidad desde la cercanía y no tuvieran una visión de oficina; y por último que tuvieran la suficiente capacidad de abstracción y una visión amplia sobre la realidad, sobre todo que hubieran desempeñado (o estén) algún tipo de trabajo de coordinación o dirección ejecutiva por la visión general que suelen dar estos puestos.

En ese primer grupo de discusión también se determinó la representatividad de los sujetos en exclusión. Fruto de este diálogo se consensuaron los siguientes grupos, como los que respondían mejor al prototipo de exclusión de espacio urbano que se estaba analizando: personas que sufrieran o hubieran sufrido pobreza económica durante un largo periodo de sus vidas; adictos, ex adictos y familiares; dis- capacitados y discapacitados de segundo orden; personas con baja cualificación; personas con alguna enfermedad estigmatizada socialmente; personas que hubieran sufrido la violencia de género; indígenas y personas de color oscuro; y personas con una orientación sexual no heterosexual. En todos los casos se trató de buscar personas que cumplieran con la variedad de criterios en lo referente a: sexo, edad, estado civil, número de dependientes, lugar de residencia y origen.

No obstante, se tuvo en cuenta en todo momento que, al ser un fenómeno dinámico y cambiante, no se podía establecer un patrón fijo de colectivos excluidos ni de factores de exclusión. La exclusión no es una característica que adquieren determinadas personas, sino que es una situación social a la que se puede llegar por múltiples trayectorias y de la que se puede salir.

En un segundo momento, central en la investigación, se ahondó en los relatos biográficos de las personas en situación de exclusión-vulnerabilidad, o que lo hubieran estado en algún momento considerable de su vida, a través de la realización de entrevistas en profundidad en pro de la realización de sus historias de vida. El fin de esta segunda fase era determinar las di- 
ferentes trayectorias vitales, las estrategias de vida y la red de apoyos familiares, sociales y gubernamentales de que dispone la persona.

En una tercera fase se hizo una recopilación exhaustiva de toda información obtenida, se clasificó y se analizó en profundidad. Posteriormente se compartieron los primeros resultados con los informantes cualificados en un segundo grupo de discusión para obtener una retroalimentación más profunda, establecer las diferentes tipologías de trayectorias de exclusión y concluir con los aprendizajes derivados del proceso de investigación, aspectos que serán ampliamente señalados a continuación.

\section{La dinámica de la exclusión social: pro- cesos y trayectorias vitales}

\subsection{Tipología de trayectorias de exclusión y puntos de inflexión}

Analizando en profundidad cada uno de los tipos de trayectorias vitales de los 17 entrevistados se agruparon en 9 tipos. Se apunta a continuación el nombre de cada uno de los tipos y se adjunta a cada uno el Gráfico 1 que sirve como modelo representativo de los mismos ${ }^{1}$.

En cada tipo se puede ver representada la trayectoria vital de los entrevistados según el espacio social en el que estuvieron en cada una de las etapas de sus vidas.

Es importante, en todo análisis sobre exclusión social, analizar los puntos de inflexión como aquellos momentos en la trayectoria vital de las personas cuando coinciden uno o más acontecimientos de gran trascendencia, tanto positivos-ascendentes como negativos-descendentes. Es interesante visualizar las situaciones acaecidas en los tipos que van del 5 al 9, donde se da una caída brusca a situaciones de vulnerabilidad o exclusión; o bien visualizar el tipo 3 , donde se da un punto de inflexión ascendente. La acumulación de ventajas (factores positivos) o desventajas sociales (factores negativos) a lo largo del tiempo, o en un momento determinado, además de la presencia de elementos desencadenantes o de un factor súbito de gran carga excluyente, conlleva el descenso o mantenimiento en una situación de vulnerabilidad o exclusión social.

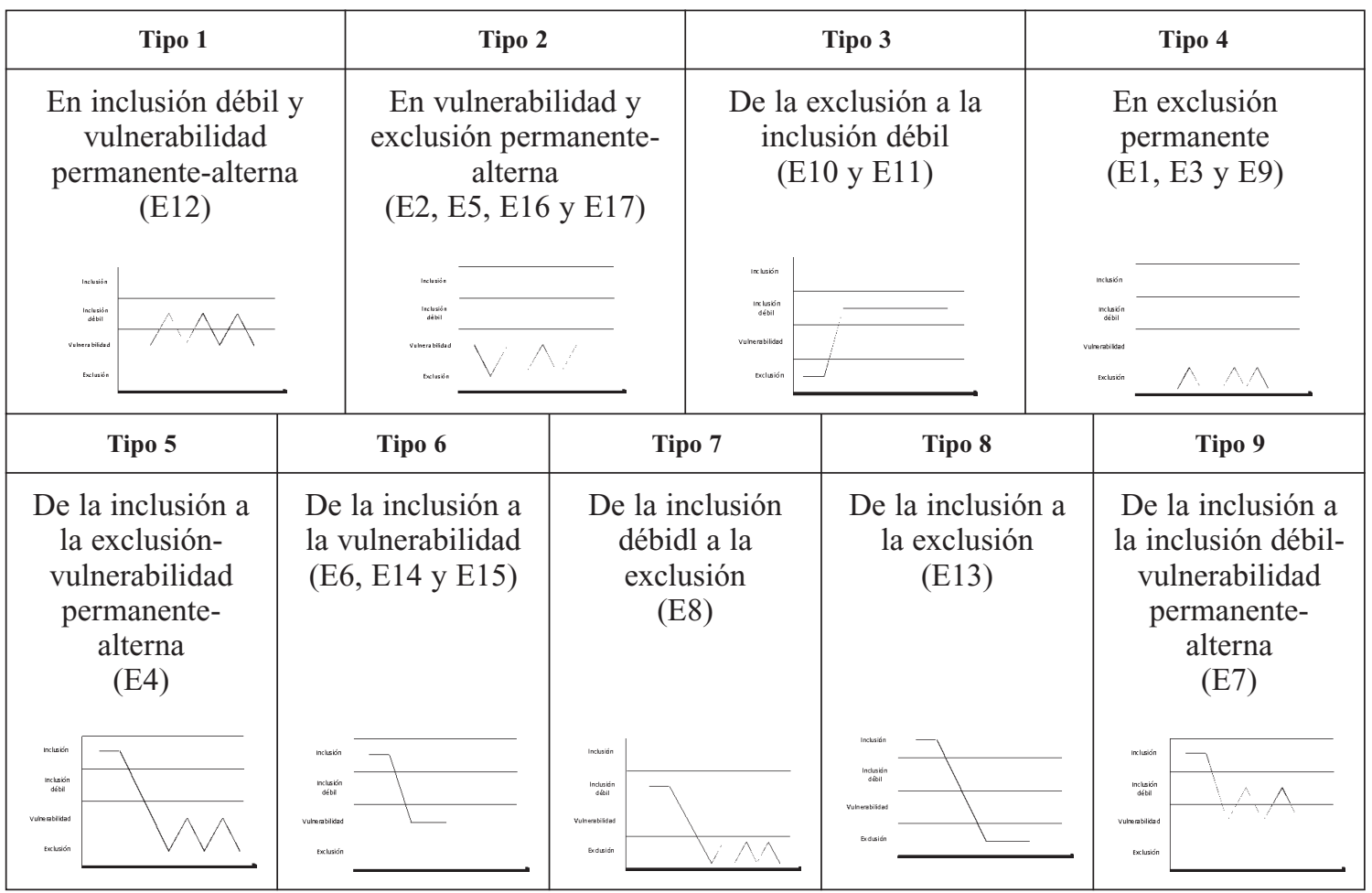

Gráfico 1.

${ }^{1}$ Se apunta en cada tipo el número y la letra de identificación de cada Entrevistado —E1 a E17-). 
Entre los factores negativos más destacados que determinan puntos de inflexión de carga exclusógena nos encontramos con los siguientes: en el ámbito familiar destacan el deterioro de las redes familiares por rupturas (divorciosseparaciones) o el abandono de uno de los miembros del núcleo familiar; las relaciones familiares conflictivas; la reproducción de las desigualdades de género en el seno de las familias; la reproducción en la familia de las situaciones de pobreza y exclusión social; el alto número de dependientes, unido a una fuente de ingresos limitada; el abandono repentino del hombre de su pareja e hijos; los altos grados de violencia intrafamiliar, al igual que la violencia en la educación de los hijos; la existencia de algún miembro con discapacidad; la alta incidencia de adicciones, especialmente alcoholismo y drogadicción, aunado a la alta tolerancia de las patologías sociales.

En cuanto al género se puede destacar que es un agravante de las situaciones de pobreza o exclusión; el papel social subordinado asignado por la organización patriarcal de la sociedad; la interiorización de la ética del cuidado de las personas dependientes por parte de la mujer, bien sean descendientes directos, ascendientes o familiares, lo que limita sus posibilidades laborales y la segmentación de las trayectorias laborales; la duplicidad y sobrecarga de trabajo; la división sexual del trabajo; la violencia de género, especialmente el maltrato psicológico y físico; el no cuestionamiento de las desigualdades de género; y el autoritarismo y superioridad masculina socialmente extendidas.

En el aspecto social y político-institucional destaca la discriminación hacia los grupos domésticos móviles; la discriminación hacia las familias con miembros problemáticos (adictos $\mathrm{y}$ violentos principalmente); el aislamiento por estigmas; la debilidad de las redes por continuas crisis, lo que obstaculiza el acceso a los recursos y espacios de socialización más valorados; los altos niveles de desconfianza hacia las instituciones públicas, autoridades, sindicatos y líderes comunitarios; los altos grados de corrupción pública; el rechazo hacia los «diferentes» (por inclinación sexual, origen étnico y religión, principalmente); la discriminación en el mundo laboral por edad, sexo, color de la piel y discapacidad; la visión paternalista y redistributiva de la justicia social; la doble moral (pública y privada); la no participación generalizada en asociaciones o grupo; y la escasez o debilidad de los servicios de apoyo social, siendo mayormente asistencialistas y paternalistas.

\subsection{Análisis de la trayectoria según el tiem- po recorrido y la forma}

Una vez clasificados cada uno de los entrevistados en las nueve tipos de trayectoria, se hizo un doble análisis de los tipos de trayectoria de exclusión social basándose en la propuesta del equipo IESA-CSIC (2010), según el tiempo de duración del recorrido y la forma. Según la duración de las trayectorias existen dos tipos: trayectorias cortas y trayectorias largas. Según la forma existen trayectorias ascendentes, trayectorias con altibajos y trayectorias descendentes (véase Figura 4).

De esta sencilla matriz de análisis se puede concluir que, las personas con trayectorias más largas se sitúan en espacios sociales de exclusión y vulnerabilidad. Las personas con trayectorias cortas se sitúan preferentemente en espacios sociales de vulnerabilidad e inclusión débil.

\section{Principales aprendizajes}

Adicionalmente a las ideas destacadas en los dos epígrafes anteriores cabe remarcar una serie de aprendizajes generales. La interrelación de los múltiples factores que generan los procesos de exclusión es compleja; evidenciando que su origen se vincula tanto con aspectos personales como institucionales, estructurales y sociales, además de que varía según las etapas de la vida, el entorno familiar, el contexto social y el apoyo de las políticas públicas.

Cuando los procesos de exclusión están enraizados en la estigmatización de identidades (por orientación sexual, origen étnico, color de la piel, colonia donde se vive y antecedentes familiares y personales, principalmente), la integración en las instituciones de la sociedad es más difícil, aunque las condiciones materiales de la persona no sean tan precarias.

Algunos de estos procesos no son evidentes, pueden estar ocultos y actúan sin que los ciudadanos sean conscientes. La exclusión social no siempre es visible, sobre todo cuando no lleva aparejados problemas de conflicto social abierto. Hay ciertos tipos de exclusión (por enfermedad estigmatizada, por discapacidad, por 


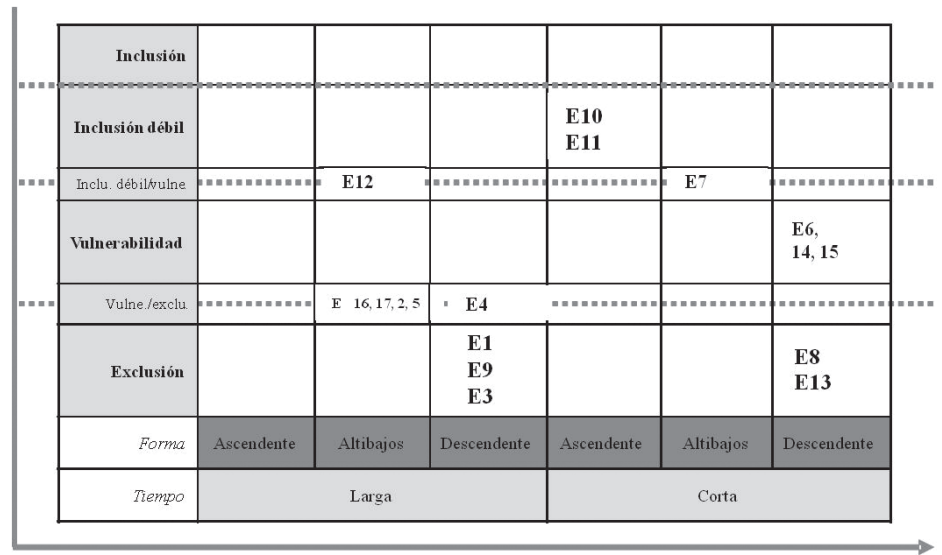

Figura 4. Cruce entre tipos de trayectorias según tiempo y forma.

Fuente: Elaboración propia.

origen étnico, por orientación sexual, principalmente) que se distribuyen por todo el territorio de la ciudad y, por lo tanto, no son fáciles de detectar.

También se ha verificado que el factor tiempo es determinante en los procesos de exclusión. Su efecto negativo se pone de manifiesto en el proceso acumulativo de desventajas sociales, pudiendo provocar situaciones crónicas de exclusión o descenso a ésta desde la vulnerabilidad. Cuanto más tiempo permanece una persona en condiciones de vulnerabilidad o exclusión más difícil se hace desarrollar estrategias de salida de su situación.

Debido a las transformaciones tan complejas que ha sufrido la sociedad han aparecido nuevos riesgos sociales o han transformado los que ya existían. El mundo de hoy demanda de mayores niveles de formación para la inserción en el mundo laboral, mayores niveles económicos para cubrir las necesidades básicas, una mayor solidez en las redes de protección sociofamiliar, por lo que, en el caso de aquellas personas que no han desarrollado estas capacidades, se encuentran en una situación cada vez más alejada de los espacios de inclusión.

$\mathrm{Al}$ respecto los dos ámbitos que tuvieron un mayor sentido explicativo fueron el relacional y el político-institucional. En el ámbito relacional los factores de mayor peso fueron el del deterioro de las redes sociales y el de las redes familiares, especialmente en lo que se refiere a los problemas familiares, ya fueran heredados o adquiridos, debido por una parte a las crisis continuas y por la otra a la agudización de los problemas sociales. Las barreras culturales y simbólicas contribuyen y refuerzan la marginalización socioeconómica y material y, en casos concretos, tienen un peso y valor mucho más fuerte en determinar la exclusión.

Entre todos los factores potenciadores de exclusión (etario, étnico, origen, formativo, orientación sexual, género, entre los principales que se han detectado en el estudio), el género es el más importante a la hora de determinar la vulnerabilidad y la exclusión social.

La familia es uno de los principales apoyos, pero a la vez también sufre las consecuencias de la exclusión si alguno de sus miembros se halla en esta circunstancia, siendo también aislada o excluida en ocasiones de la dinámica social y comunitaria, convirtiéndose así en lo que se podrían llamar "excluidos de segundo orden», es decir, personas que sin tener un factor de exclusión, sufren consecuencias parecidas a las personas en situación directa de vulnerabilidad o exclusión.

En lo referente al ámbito político-institucional, el factor que más destacó fue el de la limitación evidente de los derechos de ciudadanía de las personas en situación de vulnerabilidad o exclusión social, además de la falta de respeto y discriminación que han sufrido a lo largo de su vida. Sigue primando el paternalismo y la dependencia en las instituciones públicas y en las Organizaciones de la Sociedad Civil, sobre el empoderamiento y el desarrollo social integral. Incluso se reporta una falta de voluntad política para que se den estos cambios, por intereses partidistas y electorales. 
Las políticas de protección pública son inadecuadas, limitadas e insuficientes para el escenario social actual en materia de vivienda, empleo, asistencia sanitaria, cuidado de los niños y ancianos, comunicaciones, etc. Existe una especie de agotamiento del sistema de ayuda y desarrollo, por lo que es necesario reformularlo.

\subsection{Aprendizajes específicos sobre la reali- dad de la exclusión en el Área Metropolita- na de Monterrey}

Los aprendizajes generales quedarían incompletos si no se hiciera una referencia explícita a las características excluyentes que los entrevistados y los informantes cualificados enfatizaron como específicas de esta área urbana. Al respecto se reporta como una ciudad con unos niveles socioeconómicos muy marcados, diferenciados e impermeables, lo que conlleva que las relaciones se den prácticamente de manera exclusiva al interno de los diferentes estratos sociales. El criterio que marca el estilo de vivir, lo aceptable, está determinado por una minoría con poder. Los patrones sociales son muy predecibles. Los roles de control social están muy marcados en todos los niveles sociales y culturales: la edad para casarse, el tener hijos pronto, la mujer al servicio del hombre, la visión del hombre proveedor, la mujer al cuidado del hogar, etc.

Ciertas formas de inclusión se pueden convertir en elementos de control social por parte de la sociedad y limitan las opciones de cómo y dónde vivir las vidas. Se corre el peligro de adoptar las normas, valores y comportamientos que determinan la inclusión, aunque éstas sean profundamente excluyentes y vayan en contra de la visión personal de la vida. Pareciera como si el prototipo social de inclusión estuviera claro y quien quiere se «ajusta» a él y quien no lo haga queda excluido, en lugar de hacer un esfuerzo social de ampliación de esos espacios. Muchas personas, una vez «aceptadas» en este espacio de inclusión, adoptan los patrones y códigos excluyentes. El excluido se convierte en incluido y en excluyente.

El sentir general de la población regiomontana es el de que son gente trabajadora, honesta y luchadora. Los antecedentes industriales crearon una suerte de cultura del trabajo muy arraigada en la población. Ese sentir se ha reportado como un aspecto muy positivo de la población de esta urbe. Sin embargo, como contracara, se explicita que ésta, en ocasiones, se puede llegar a convertir en una cultura de la superioridad, a la vez que hedonista y materialista, lo que lleva a un rechazo de los más pobres, marginados y excluidos, catalogándolos y estigmatizándolos como los culpables de su situación porque no quieren salir adelante, sin alcanzar a visualizar que hay otras causas de origen cultural-simbólico que en muchas ocasiones tiene mayor fuerza y que dependen de la misma dinámica social.

Al ser reconocida tradicionalmente como una ciudad industrial, aunque actualmente el sector más predominante sea el de servicios, las personas son clasificadas en función de su utilidad: vales si eres productivo. La persona que no está inmersa en los círculos laborales o no tiene la posibilidad de hacerlo por los estigmas sociales o por sus discapacidades, es socialmente vista como inferior o de otra categoría. La cultura del trabajo deriva en competitividad.

Esta cultura de la superioridad lleva a la necesidad de distinguirse de los demás por los bienes y la apariencia, lo que ha hecho que se desarrollen toda una serie de códigos que determinan la aceptación-inclusión social. Incluso ha llegado a desarrollarse un imaginario colectivo de ser lo más parecido al primer mundo, estando así conectada a otras regiones del mundo por un lado, y desconectada a las realidades interiores más crudas por el otro. Todo lo que se ajuste a este imaginario forma parte de los elementos de inclusión de la sociedad; lo que no se ajusta a este prototipo queda excluido y marginado.

Existe igualmente una falsa creencia de que en Monterrey se puede hacer dinero fácilmente si se quiere trabajar. La cuestión es que, actualmente, las fuentes de empleo son cada vez más escasas, el mercado laboral está cada vez más polarizado, y el acceso a las oportunidades de los mejores trabajos está limitado. Monterrey ya no da posibilidades de crecer a la población en general. Sigue siendo una ciudad de muy buenas oportunidades, pero solo para unos pocos.

Uno de los aspectos destacados en el análisis es el religioso: quien no profesa la religión católica es mal visto. Prácticamente la unanimidad de los entrevistados reportan que la sociedad es más moralista que religiosa, centrada en preceptos y cumplimientos, más que en 
compromisos y vivencia de la fe. Estas prácticas moralistas son las que marcan, en muchos casos, el «deber ser» social.

Este hecho está determinado por una doble moral, donde se manifiesta una cara pública conversadora, y una cara privada en donde todo vale y se puede. Esta doble moral es la que reportan los excluidos como una de las que más les afecta en determinados casos, pues aunque algunos de ellos son rechazados públicamente, en lo privado son aceptados o respetados.

Existe poca tolerancia y respeto a los «diferentes». Se anula la diversidad urbana. Las minorías no tienen poder ni son reconocidas socialmente. Muchas subculturas urbanas existen, pero apenas son visibles o bien son invisibilizadas. Hay miedo al diferente porque se puede convertir en amenaza a los cotos de poder y al control social.

\section{Conclusión}

Aunque todo el epígrafe anterior podría considerarse como una gran conclusión cabe recalcar, como cierre del artículo, que para superar las dinámicas de exclusión es necesario trabajar cambios en la sociedad en conjunto, de manera directa y específica con los incluidos, pues son los que establecen y controlan las fronteras-barreras materiales, sociales, culturales y simbólicas. Se debe poner el acento en las características sociales que excluyen, para así también trabajar en esa dimensión, ya que de poco servirá empoderar a la persona y capacitarla, si luego va a sentir el bloqueo del acceso a los espacios socialmente más valorados de cara el desarrollo. Es necesaria la acción e intervención conjunta en toda la sociedad tanto en la excluida como en la excluyente.

Los programas centrados en los resultados tienden a enfocarse en soluciones remediales, tratando de mejorar sobre todo los ingresos y facilitando el acceso al mundo laboral y a los servicios, pero es una visión limitada, ya que cada tipo de exclusión requiere una respuesta diferente. Centrarse en los aspectos socioeconómicos y materiales es trabajar con las consecuencias y, por lo tanto, es una intervención remedial. Ir a la raíz y al origen de muchos problemas es centrarse en parte en la exclusión cultural simbólica y, por lo tanto, las respuestas tienen que ser transformativas de la sociedad en conjunto.

Así pues, es fundamental no centrarse únicamente en proyectos tradicionales de transferencia de renta o incremento de cualificación sobre todo para el empleo. Aunado a esto tiene que haber políticas de integración, ya que de nada sirve capacitar y desarrollar a una persona si no se incide en las estructuras que crean las fronteras sociales de inclusión. El foco de las políticas sociales no puede estar centrado únicamente en incentivar el crecimiento económico y la incorporación al mercado de trabajo, como la base para erradicar la pobreza y fomentar la cohesión social.

Si bien la combinación de factores es específica para cada persona o grupo y da lugar a situaciones concretas de exclusión muy diferenciadas -siendo necesario por lo tanto usar una matriz personal de exclusión- es importante recalcar que todo itinerario de exclusión muestra una compleja interrelación de múltiples factores que generan los procesos de exclusión; evidenciando que su origen se vincula tanto con aspectos personales como institucionales, estructurales y sociales, además de que varía según las etapas de la vida, el entorno familiar, el contexto social y la existencia de políticas públicas que generen un verdadero impacto y cambio social.

\section{Referencias bibliográficas}

Brambila, C. (2004). La pobreza urbana en México. (Documento de trabajo para el informe sobre Desarrollo Humano México 2004 «El reto del desarrollo local»). Ciudad de México: Tecnológico de Monterrey.

Castel, R. (1997). La metamorfosis de la cuestión social: una crónica del salariado, Buenos Aires: Paidós.

Gacitúa, E., Sojo, C. y Davis, S (eds.) (2000). Exclusión social y reducción de la pobreza en América Latina y El Caribe. San José C.R.: FLACSO y Banco Mundial.

Hernández Pedreño, M. (1999). Tiempos de vejez, Murcia: Universidad de Murcia.

Hernández Pedreño, M. (dir.) (2008). Exclusión social en la región de Murcia. Aproximación cuantitativa y cualitativa, Murcia: Editum. 
Instituto Catalán de Gobierno y Políticas Públicas. (2010). Ciutadania i Inclusió Social. El Tercer Sector y les politiques publiques d'acció social (Document per al debat 4.). Barcelona: Universitat Autònoma de Barcelona y Fundació Catalana de 1'Esplai.

Instituto de Estudios Sociales Avanzados del Consejo Superior de Investigaciones Científicas. (2010). Diagnóstico sobre las condiciones de vida de la población en Córdoba. Procesos de Exclusión. Ayuntamiento de Córdoba 2009-2010. Córdoba: Ayuntamiento de Córdoba.

Mira Albert, S. (2008). Indicadores sociales y valoración de la exclusión. En M. Hernández Pedreño (coord.), Exclusión social y desigualdad. Murcia: Editum.

Olaz Capitán, A. (2008). La entrevista en profundidad. Oviedo: Septem ediciones.

Pérez Yruela, M., Rodríguez Cabrero, G. y Trujillo Carmona, M. (2004). Pobreza y exclusión social en el Principado de Asturias. Córdoba: CSIC?IESA.

Pérez Yruela, M., Sáez Méndez, H. y Trujillo Carmona, M. (2002). Pobreza y exclusión social en Andalucía. Córdoba: CSIC?IESA.

Raya Díez, E. (2004). Indicadores de exclusión social. Una aproximación al estudio aplicado de la exclusión. Bilbao: Universidad del País Vasco.

Rubio, M.J. y Montero, S. (coords.) (2002). La exclusión social. Teoría y práctica de la intervención. Madrid: CCS.

Ruiz Olabuénaga, J. L. (2007). Metodología de la investigación social. Bilbao: Universidad de Deusto.

Subirats, J. y Gomà, R. (dirs.) (2003). Un paso más hacia la inclusión social. Generación de conocimiento, políticas y prácticas para la inclusión social. Madrid: Instituto de Gobierno y Políticas Públicas; Barcelona: Universidad Autónoma de Barcelona.

Subirats, J., Gomà, R. y Brugué, J. (coords.) (2005). Análisis de los factores de exclusión social. Bilbao: Fundación BBVA; Barcelona: Institut d'Estudis Autonòmics, Generalitat de Catalunya.

Tilly, Ch. (1999). Durable inequality. California: University of California Press.

Ward, N. (abril, 2009). Social exclusion, social identity and social work: Analysing social exclusion from a material discursive perspective. Social Work Education, 28 (3), 237-252. 